\title{
Tiempo óptimo para medición de paratohormona como predictor de hipocalcemia en pacientes posterior a tiroidectomía total
}

\author{
Optimal time for measurement of parathormone as a predictor of \\ hypocalcemia in patients after total thyroidectomy
}

\author{
Jenith $\operatorname{Lagos}^{1} \mathbb{D}$, Jorge A. Herrera² $\mathbb{D}$, Daniela $\operatorname{Lagos}^{3} \mathbb{D}$, Ángela Merchán-Galvis ${ }^{4} \mathbb{D}$
}

\begin{abstract}
Médica, especialista en Cirugía General, Fellow de Cirugía Vascular, Universidad de Antioquia, Medellín, Colombia.
2 MD, FACS, especialista en Cirugía General, Miembro del Latin American Thyroid Society; Director Científico, Clínica La Estancia; profesor, Cirugía general, Facultad Ciencias de la Salud, Universidad del Cauca, Popayán, Colombia.

Estudiante, programa de Medicina, Universidad del Cauca, Popayán, Colombia.

4 MD, MSc, magíster en Epidemiología Clínica, Profesora Departamento de Medicina Social y Salud Familiar, Facultad Ciencias de la Salud, Universidad del Cauca, Popayán, Colombia.
\end{abstract}

\section{Resumen}

Introducción. La hipocalcemia posterior a tiroidectomía total es una complicación frecuente en la cirugía de tiroides. La hormona paratiroidea ha demostrado ser un predictor confiable para detectar los pacientes con alto riesgo de hipocalcemia significativa y la consecuente necesidad de administrar suplemento de calcio. El objetivo de este estudio fue determinar el tiempo óptimo de medición de la paratohormona para dicho fin.

Métodos. Estudio prospectivo realizado en dos instituciones de nivel III en Popayán, Colombia, entre abril de 2016 y febrero de 2018, en el cual se midieron niveles de paratohormona prequirúrgico, a las 0, 2, 4 y 12 horas del postoperatorio, y se compararon con los valores obtenidos a los 3 meses de seguimiento en pacientes sometidos a tiroidectomía total. Se consideró como grupos de riesgo y con necesidad de suplemento de calcio un valor de paratohormona menor de $10 \mathrm{pg} / \mathrm{ml}$ o una disminución mayor del $80 \%$ con respecto al valor prequirúrgico.

Resultados. Se incluyeron 34 pacientes intervenidos. Tomando como umbral un valor menor de $10 \mathrm{pg} / \mathrm{ml}$, la medición de paratohormona a las 4 horas fue sensible y específica (60 y 93,1 \% respectivamente). Para una disminución mayor del $80 \%$ con respecto al valor prequirúrgico, la sensibilidad fue del 60 \% y la especificidad del $96,5 \%$, presentando un valor predictivo positivo de $75 \%$ y negativo del $93,3 \%$, con una $\mathrm{p}<0,001$.

Fecha de recibido: 05/04/2020 - Fecha de aceptación: 16/08/2020

Correspondencia: Ángela Merchán-Galvis. Carrera 6 \# 13N - 50, Universidad del Cauca, Popayán, Colombia. Teléfono: +57 3176545717 Correspondencia: angelamerchan@unicauca.edu.co

Citar como: Lagos J, Herrera JA, Lagos D, Merchán-Galvis A. Tiempo óptimo para medición de paratohormona como predictor de hipocalcemia en pacientes posterior a tiroidectomía total. Rev Colomb Cir. 2021;36:51-9. https://doi.org/10.30944/20117582.601

Este es un artículo de acceso abierto bajo una Licencia Creative Commons - BY-NC-ND https://creativecommons.org/licenses/by-ncnd/4.0/deed.es 
Discusión. Una disminución mayor del 80 \% del valor de paratohormona prequirúrgico medida a las 4 horas después de la tiroidectomía total permite identificar aquellos pacientes con alto riesgo de hipocalcemia significativa, que requieren administración temprana de suplementos de calcio, comparado con la determinación de la disminución a las 0,2 y 12 horas postoperatorias.

Palabras clave: hipocalcemia; hormona paratiroidea; hormonas y agentes reguladores de calcio; tiroidectomía; complicaciones posoperatorias; diagnóstico.

\begin{abstract}
Introduction. Hypocalcemia after total thyroidectomy is a common complication in thyroid surgery. Parathyroid hormone has been shown to be a reliable predictor for detecting patients with at high risk of significant hypocalcemia and the consequent need for calcium supplementation. The objective of this study was to determine the optimal time for measuring parathormone for this purpose.
\end{abstract}

Methods. Prospective study carried out in two level 3 institutions in Popayán, Colombia, between April 2016 and February 2018, in which levels of preoperative parathormone were measured at 0, 2, 4 and 12 hours postoperatively, and compared with the values obtained at 3 months of follow-up in patients undergoing total thyroidectomy. A parathormone value less than $10 \mathrm{pg} / \mathrm{ml}$ or a decrease greater than $80 \%$ with respect to the pre-surgical value was considered as risk groups and in need of calcium supplementation.

Results. Thirty-four operated patients were included. Taking as a threshold a value of less than $10 \mathrm{pg} / \mathrm{ml}$, the measurement of parathormone at 4 hours was sensitive and specific ( $60 \%$ and $93.1 \%$, respectively). For a decrease greater than $80 \%$ with respect to the presurgical value, the sensitivity was $60 \%$ and the specificity was $96.5 \%$, presenting a positive predictive value of $75 \%$ and a negative predictive value of $93.3 \%(\mathrm{p}<0.001)$.

Discussion. A decrease of more than $80 \%$ in the preoperative parathyroid hormone value measured at 4 hours after total thyroidectomy allows the identification of those patients at high risk of significant hypocalcaemia, who require early administration of calcium supplements, compared with the determination of the decrease at 0,2 and 12 hours postoperatively.

Keywords: hypocalcemia; parathyroid hormone; calcium-regulating hormones and agents; thyroidectomy; postoperative complications; diagnosis.

\section{Introducción}

La hipocalcemia transitoria posterior a tiroidectomía total es la complicación más frecuente de la cirugía de tiroides, observándose en un 1,6 - $68 \%$ de los pacientes ${ }^{1-3}$. En su mayoría es debida a compromiso vascular y en menor proporción a la resección inadvertida de las paratiroides ${ }^{4-8}$. Su presentación clínica varía en un amplio rango, desde pacientes asintomáticos hasta la tetania y el shock ${ }^{5,9-11}$. Dada la incertidumbre sobre la evolución de los niveles de calcio después de tiroidectomía total, los pacientes requieren estudios adicionales, que prolongan su hospitalización, o suplementos con calcio de forma sistemática.
Aunque, la hipocalcemia suele ser transitoria, usualmente menor a 6 meses, en $1-10 \%$ de los casos puede ser permanente ${ }^{6,7,9,12}$. La identificación de los pacientes con riesgo de hipocalcemia transitoria significativa se traduce en disminución de la morbilidad, costos y necesidad de estancia hospitalaria prolongada, obviando la administración innecesaria de suplementos de calcio ${ }^{11}$. De allí que los niveles de calcio postoperatorio, aunque son importantes como predictor de hipocalcemia sintomática, sólo son relevantes después de 24 a 48 horas ${ }^{9,13}$.

La hormona paratiroidea (PTH), o paratohormona, es regulada de forma primaria por los nive- 
les séricos de calcio ionizado y, una vez secretada, se elimina rápidamente del plasma, con una vida media de dos a cuatro minutos ${ }^{14,15}$. Basados en lo anterior, múltiples estudios avalan la medición de la PTH como una prueba útil para la detección de los pacientes con alto riesgo de hipocalcemia posterior a una cirugía de tiroides; sin embargo, hay heterogeneidad sobre los métodos y la hora idónea para realizar la prueba ${ }^{2,4,7,12,13,15-31}$.

El objetivo de este estudio fue determinar el tiempo óptimo para la medición de la PTH, como predictor de hipocalcemia, en pacientes sometidos a tiroidectomía total.

\section{Métodos}

Este fue un estudio prospectivo de prueba diagnóstica, que incluyó pacientes mayores de 18 años a quienes se les practicó una tiroidectomía total, en el Hospital Universitario San José y Clínica La Estancia, instituciones de nivel III de la ciudad de Popayán, Colombia. Se excluyeron los pacientes con alteraciones previas de los niveles de PTH o calcio, patología paratiroidea, insuficiencia renal o tratamientos farmacológicos que intervienen con los niveles de calcio; así como aquellos que no cumplieron con la totalidad de muestras solicitadas para el estudio. Se aplicó muestreo no probabilístico por conveniencia.

El proyecto se clasificó como una investigación de bajo riesgo y contó con la aprobación de los comités de ética institucional, respetando y protegiendo los principios bioéticos de la investigación en humanos, siguiendo lo establecido en la Declaración de Helsinki y la Resolución No. 008430 de 1993 de Colombia. Se obtuvo el consentimiento informado de todos los pacientes incluidos y sus datos fueron tratados acorde a Ley 1581 de 2012 y Decreto 1377 de 2013.

Se recogió información sobre las características sociodemográficas y clínicas, se midieron niveles de calcio ionizado prequirúrgico y a las 12 horas después de la cirugía, tomando como valores en rango de normalidad aquellos que se encuentran entre 1,12 - 1,33 mmol/L. Se tomaron los niveles de PTH en el prequirúrgico, al cierre de la incisión quirúrgica (0 horas), 2, 4 y 12 horas del postquirúrgico, así como una muestra de control a los 3 meses. El análisis de la PTH se procesó en equipo LIAISON ${ }^{\circledR}$ N-TACT ${ }^{\circledR}$ PTH Gen 11 por inmunoensayo quimioluminiscente in vitro, tomando como niveles normales valores entre 22,2 - $108,9 \mathrm{pg} / \mathrm{ml}$. De igual forma se documentaron los hallazgos en el intraoperatorio, hospitalización, manifestaciones clínicas de hipocalcemia y necesidad de suplementación de calcio, en un seguimiento hasta el día posterior a la intervención.

Los datos obtenidos se analizaron usando el paquete estadístico SPSS versión 25. Para la descripción de la población se emplearon medidas de tendencia central y de dispersión en las variables cuantitativas, así como frecuencias y proporciones en las variables categóricas. Se dividió a la población en pacientes de alto riesgo de hipocalcemia, si la PTH fue menor a $10 \mathrm{pg} / \mathrm{ml}$ o con un descenso mayor al $80 \%$ con respecto al nivel basal, para cada hora definida y se estimó sensibilidad, especificidad y valores predictivos positivo y negativo (VPP y VPN), para determinar el momento idóneo para la toma de la prueba. El nivel de significación estadística se estableció en $\mathrm{p}<0,05$.

\section{Resultados}

Se intervinieron 43 pacientes en el periodo comprendido entre abril de 2016 y febrero de 2018, de los cuales 9 se excluyeron por no cumplir con el número requerido de muestras. Un total de 34 pacientes se admitieron para el estudio. La media de la edad fue de 47,7 años y la desviación estándar (SD) de 16,9 (rango: 19-83 años); el 91,2\% eran mujeres $(\mathrm{n}=31)$, predominó la etnia mestiza $82,4 \%(n=28)$ y un $58,8 \%$ vivía en pareja $(n=20)$ (tabla 1). El $66 \%(n=19)$ de los pacientes tenía comorbilidades, siendo las del sistema cardiovascular las más frecuentes (23,5\%), seguida de las endocrinas $(20,6 \%)$.

La indicación de tiroidectomía total fue dada por histología maligna, obtenida por biopsia en un $61,8 \%(n=21)$, siendo el carcinoma papilar la principal causa. Por otra parte, aquellos pacientes cuyo reporte histopatológico de biopsia no fue conclusivo $(n=18 ; 23,5 \%)$, fueron operados a criterio del cirujano tratante.

En el 79,4 \% de los pacientes ( $n=27)$, el procedimiento fue tiroidectomía total, y en el resto se 
Tabla 1. Caracterización sociodemográfica y clínica de la población estudio.

\begin{tabular}{|c|c|c|c|}
\hline \multicolumn{2}{|c|}{ Variable Sociodemográficas } & \multirow{2}{*}{$\begin{array}{c}\mathbf{n} \\
31\end{array}$} & \multirow{2}{*}{$\begin{array}{c}\% \\
91,2\end{array}$} \\
\hline Cóngr & Femenino & & \\
\hline & Masculino & 3 & 8,8 \\
\hline \multirow{3}{*}{ Edad } & Media & \multicolumn{2}{|c|}{47,7} \\
\hline & Desviación estándar & \multicolumn{2}{|c|}{16,9} \\
\hline & Rango & \multicolumn{2}{|c|}{$19-83$} \\
\hline \multirow{3}{*}{ Etnia } & Mestizo & 28 & 82,4 \\
\hline & Indígena & 3 & 8,8 \\
\hline & Negro & 3 & 8,8 \\
\hline \multirow{4}{*}{ Estado civil } & Casado/Unión libre & 20 & 58,8 \\
\hline & Soltero & 10 & 29,4 \\
\hline & Separado/divorciado & 2 & 5,9 \\
\hline & Viudo & 2 & 5,9 \\
\hline \multirow{2}{*}{ Procedencia } & Rural & 16 & 47,1 \\
\hline & Urbana & 18 & 52,9 \\
\hline \multirow{5}{*}{ Nivel educativo } & Ninguno & 4 & 11,8 \\
\hline & Primaria & 10 & 29,4 \\
\hline & Bachillerato & 7 & 20,6 \\
\hline & Tecnológico & 9 & 26,5 \\
\hline & Universitario & 4 & 11,8 \\
\hline \multicolumn{2}{|c|}{ Variables Clínicas } & $\mathbf{n}$ & $\%$ \\
\hline \multirow{4}{*}{ Comorbilidad } & Ninguna & 15 & 44,1 \\
\hline & Cardiovascular & 8 & 23,5 \\
\hline & Endocrina & 7 & 20,6 \\
\hline & $\begin{array}{l}\text { Endocrina y Cardiovas- } \\
\text { cular }\end{array}$ & 4 & 11,8 \\
\hline \multirow{3}{*}{$\begin{array}{l}\text { Diámetro } \\
\text { prequirúrgico } \\
\text { de la lesión }\end{array}$} & Menor de $2 \mathrm{~cm}$ & 10 & 35,7 \\
\hline & $2-4 \mathrm{~cm}$ & 10 & 35,7 \\
\hline & Mayor de $4 \mathrm{~cm}$ & 8 & 28,6 \\
\hline \multirow{3}{*}{$\begin{array}{l}\text { Patología } \\
\text { prequirúrgica }\end{array}$} & Maligna & 21 & 61,8 \\
\hline & Patología no conclusiva & 8 & 23,5 \\
\hline & Benigna & 5 & 14,7 \\
\hline \multirow{4}{*}{ Histología final } & Neoplasia papilar & 19 & 55,8 \\
\hline & Neoplasia folicular & 2 & 5,9 \\
\hline & Bocio multinodular & 11 & 32,4 \\
\hline & Tiroiditis & 2 & 5,9 \\
\hline
\end{tabular}

adicionó vaciamiento ganglionar, uni o bilateral (tabla 2). El 67,6 \% (n=23) no presentó extensión extra-tiroidea. En el 55,9\% se preservaron 4 paratiroides $(n=19)$ y no se realizó autotransplante. La histología final reportó patología maligna en el $61,8 \%(n=21)$, predominando el carcinoma papilar.

La estancia media hospitalaria fue de 28 horas, con una mediana de 25 horas y un rango de 13-72 horas. Se presentaron síntomas leves de hipocalcemia en dos pacientes del estudio, correspondientes al 5,9\% de los casos (uno de ellos presentó niveles bajos de PTH desde la cirugía hasta el último control), y no se reportaron eventos graves de hipocalcemia que requirieran uso de calcio intravenoso.

Los niveles calcio ionizado y PTH, y el porcentaje de disminución de PTH se presentan en la tabla 3. La frecuencia de niveles de PTH menores a 22,2 $\mathrm{pg} / \mathrm{ml}$ a las $0,2,4$ y 12 horas fue de $73,5 \%(\mathrm{n}=25)$, $67,6 \%(n=23), 55,8 \%(n=19)$ y $44,1 \%(n=15)$, respectivamente. Con el pasar de las horas, los

Tabla 2. Características del procedimiento quirúrgico realizado.

\begin{tabular}{|c|c|c|c|}
\hline \multicolumn{2}{|c|}{ Descripción del procedimiento } & \multirow{2}{*}{$\begin{array}{c}\mathbf{n} \\
27\end{array}$} & \multirow{2}{*}{$\begin{array}{c}\% \\
79,4\end{array}$} \\
\hline \multirow[b]{2}{*}{ Tipo de Cirugía } & Tiroidectomía total & & \\
\hline & $\begin{array}{l}\text { Tiroidectomía total más } \\
\text { vaciamiento }\end{array}$ & 7 & 20,6 \\
\hline \multirow{2}{*}{ Tipo de incisión } & Transversa estándar & 25 & 73,5 \\
\hline & Transversa ampliada & 9 & 26,5 \\
\hline \multirow{2}{*}{$\begin{array}{l}\text { Extensión extra } \\
\text { tiroidea }\end{array}$} & No & 23 & 67,6 \\
\hline & Si & 11 & 32,4 \\
\hline \multirow{2}{*}{ Adenopatías } & No & 29 & 85,3 \\
\hline & $\mathrm{Si}$ & 5 & 14,7 \\
\hline \multirow{3}{*}{$\begin{array}{l}\text { Número de } \\
\text { paratiroides } \\
\text { preservadas }\end{array}$} & 2 & 4 & 11,8 \\
\hline & 3 & 11 & 32,3 \\
\hline & 4 & 19 & 55,9 \\
\hline \multirow{3}{*}{$\begin{array}{l}\text { Tiempo quirúrgico } \\
\text { (minutos) }\end{array}$} & Media & \multicolumn{2}{|c|}{106} \\
\hline & Desviación estándar & \multicolumn{2}{|c|}{33} \\
\hline & Rango & \multicolumn{2}{|c|}{$70-180$} \\
\hline \multirow{3}{*}{$\begin{array}{l}\text { Estancia } \\
\text { hospitalaria (horas) }\end{array}$} & Media & \multicolumn{2}{|c|}{28,4} \\
\hline & Desviación estándar & \multicolumn{2}{|c|}{11,1} \\
\hline & Rango & \multicolumn{2}{|c|}{$13-72$} \\
\hline
\end{tabular}


niveles de PTH tendieron a normalizarse, pero fueron inferiores a los obtenidos en el seguimiento a los 3 meses; sin embargo no alcanzaron los valores reportados en el prequirúrgico tal como se presentan en la figura 1.

Se dio egreso al 17,6\% $(\mathrm{n}=6)$ de los pacientes con suplemento de calcio, basados en los resultados de PTH a las 12 horas (menor de $10 \mathrm{pg} / \mathrm{ml} \mathrm{o}$ disminución mayor del 80 \% con relación al valor prequirúrgico), o en la presentación de síntomas de hipocalcemia.

Utilizando una PTH sérica de $10 \mathrm{pg} / \mathrm{ml}$ como umbral para definir la necesidad de suplementos de calcio, se calculó la sensibilidad y especificidad para cada muestra tomada y se describen en la tabla 4. De acuerdo con lo observado, la PTH tomada a las 4 horas es más sensible ( $60 \%$ ) y con un valor predictivo negativo superior $(93,1 \%)$, mientras que a las 12 horas es más específica $(96,5 \%$ ) y presenta un valor predictivo positivo mayor $(66,6 \%)$. La muestra de las 4 horas fue estadísticamente significativa $(\mathrm{p}=0,01)$.

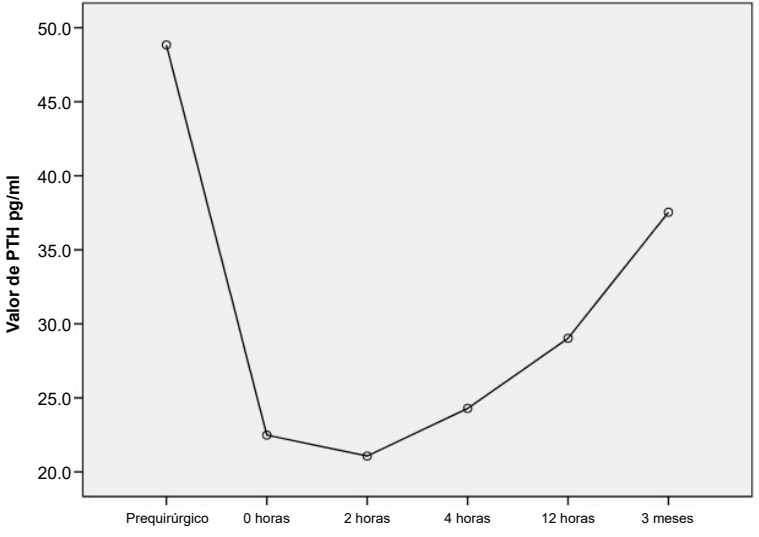

Figura 1. Evolución de niveles de PTH en pacientes llevados a tiroidectomía total.

Si se usa una disminución mayor del 80 \% del nivel basal como referencia para la identificación de los pacientes a riesgo de hipocalcemia y decidir la necesidad de suplementos de calcio en el postoperatorio, el momento considerado más sensible (60\%), específico (96,5\%), con valor

Tabla 3. Niveles pre y postoperatorios de calcio, PTH y porcentaje de disminución de PTH

\begin{tabular}{|c|c|c|c|c|c|c|c|c|c|c|c|}
\hline & \multicolumn{2}{|c|}{ Preoperatorio } & \multicolumn{2}{|c|}{ PTH 0 horas } & \multicolumn{2}{|c|}{ PTH 2 horas } & \multicolumn{2}{|c|}{ PTH 4 horas } & \multicolumn{2}{|c|}{ PTH 12 horas } & \multirow{2}{*}{$\begin{array}{c}\text { PTH } 3 \\
\text { meses } \\
\text { Valor }\end{array}$} \\
\hline & Calcio & PTH & Valor & $\%$ Dism & Valor & $\%$ Dism & Valor & $\%$ Dism & Valor & $\%$ Dism & \\
\hline Media & 1,22 & 48,83 & 22,48 & 55,47 & 21,66 & 56,25 & 24,29 & 51,34 & 29,02 & 41,09 & 37,53 \\
\hline Mediana & 1,23 & 40,70 & 15,85 & 57,57 & 17,50 & 60,11 & 20,0 & 50,58 & 22,55 & 40,34 & 29,00 \\
\hline SD & 0,04 & 24,11 & 22,06 & 25,27 & 20,07 & 25,77 & 19,31 & 21,46 & 20,41 & 24,33 & 19,92 \\
\hline Mínimo & 1,14 & 22,50 & 3,0 & 5,56 & 3,0 & 3,77 & 3,20 & 1,89 & 5,90 & $-26,51^{*}$ & 14,5 \\
\hline Máximo & 1,32 & 108,0 & 102,0 & 93,52 & 102,0 & 93,52 & 104,0 & 90,06 & 104,6 & 85,95 & 100,2 \\
\hline
\end{tabular}

*Porcentaje en negativo dado que un paciente presentó un valor postquirúrgico superior al prequirúrgico.

Tabla 4. Valor predictivo de $\mathrm{PTH}<10 \mathrm{pg} / \mathrm{ml}$ a las $0,2,4$ y 12 horas versus la presencia de niveles bajos de PTH al 3 mes postoperatorio

\begin{tabular}{|c|c|c|c|c|c|c|c|c|c|}
\hline & \multicolumn{2}{|c|}{ PTH $<10 \mathrm{pg} / \mathrm{ml}$} & \multicolumn{2}{|c|}{ PTH $>10 \mathrm{pg} / \mathrm{ml}$} & \multirow{2}{*}{ Sensibilidad } & \multirow{2}{*}{ Especificidad } & \multirow{2}{*}{ VPP \% } & \multirow{2}{*}{ VPN \% } & \multirow{2}{*}{$\mathrm{p}$} \\
\hline & $\mathrm{n}$ & $\%$ & $\mathrm{n}$ & $\%$ & & & & & \\
\hline PTH 0 horas & 6 & 17,6 & 28 & 82,4 & $40 \%$ & $86 \%$ & 33,3 & 89,2 & 0,15 \\
\hline PTH 2 horas & 8 & 23,5 & 26 & 76,5 & $40 \%$ & $79 \%$ & 25 & 88,4 & 0,34 \\
\hline PTH 4 horas & 5 & 14,7 & 29 & 85,3 & $60 \%$ & $93,1 \%$ & 60 & 93,1 & $0,01^{*}$ \\
\hline PTH 12 horas & 3 & 8,8 & 31 & 91,2 & $40 \%$ & $96,5 \%$ & 66,6 & 90 & $0,05^{*}$ \\
\hline
\end{tabular}

* Se usó prueba exacta de Fisher 
predictivo positivo (75 \%) y valor predictivo negativo $(93,3 \%)$ superiores, con una diferencia estadísticamente significativa ( $p=<0,001)$, es a las 4 horas (tabla 5).

El seguimiento a los pacientes incluidos en el estudio fue de 3 meses, momento en el que se solicitaron niveles de PTH, observando una mediana de 29,0 pg/ml (rango: 14,5-100,2 pg/ml). Se encontraron niveles bajos de PTH en el 14,7 \% $(n=5)$ de los pacientes, dos operados por patología benigna y tres por patología maligna, con mediana de PTH de 19,0 pg/ml, por lo que aún requerían suplementos de calcio y calcitriol. No se documentaron muertes durante el periodo de seguimiento.

\section{Discusión}

Este estudio, que buscó establecer el momento óptimo para la toma de $\mathrm{PTH}$, como predictor de hipocalcemia transitoria postquirúrgica en pacientes intervenidos por tiroidectomía total, tuvo una población con una edad media de 47,7 años, similar a lo descrito en la literatura ${ }^{16-20,22-27,32}$; $\sin$ embargo, nuestros pacientes tuvieron una distribución simétrica para cada una de las décadas, con solo un $20 \%$ entre la $4^{\text {a }}$ y $5^{\text {a }}$ década, a diferencia de lo descrito por el Nacional Cancer Institute, que reporta incidencias mayores entre la $4^{\mathrm{a}}$ y $5^{\mathrm{a}}$ década de la vida ${ }^{33}$, posiblemente debido a la distribución demográfica de nuestro país.

La población estudiada fue predominantemente de sexo femenino, siendo la relación hombre-mujer $1: 10$, superior a la reportada para Colombia de $1: 8^{33}$ y ligeramente superior a la descrita por otros autores ${ }^{2,13,28-30}$.
La principal indicación de tiroidectomía fue la patología maligna, siendo el carcinoma papilar la histología más frecuente, lo que coincide con los reportes de AlQahtani ${ }^{27}$, Cayo ${ }^{22}$ y Landry ${ }^{21}$, pero diferente de la mayoría de publicaciones, donde la principal causa fueron las patologías benignas $7,11-13,16,18,19,25,28-30,32$. El porcentaje de pacientes con hipocalcemia a las 12 horas fue cercano al $25 \%$, lo que se encuentra dentro del rango reportado en diversos estudios $4,7,11-13,19,20,22-$ 27,29,31,32,34.

En el primer método de clasificación utilizando en este estudio, con valores de PTH menores de $10 \mathrm{pg} / \mathrm{ml}$, los niveles a las 4 horas tuvieron sensibilidad de $60 \%$ y especificidad del $93 \%$, con VPP del 60 \% y VPN del $93 \%$, siendo esta la hora que muestra una diferencia estadísticamente significativa $(p=0,01)$. Al respecto, diversos autores ${ }^{19,25,26,29}$ reportaron que la medición de PTH entre $10-12,5 \mathrm{pg} / \mathrm{ml}$ a las 4 horas, presentaba una sensibilidad superior y una especificidad similar a la de nuestro estudio, para predecir hipocalcemia postoperatoria. Es probable que las cifras sean mayores en esos estudios retrospectivos por el mayor número de pacientes intervenidos.

Sobre el segundo método de estimación de riesgo de hipocalcemia (disminución de PTH mayor del $80 \%$ del valor basal), la medición de PTH a las 4 horas tuvo una sensibilidad del $60 \%$ y especificidad del $96,5 \%$, con VPP del $75 \%$ y VPN del 93,3\%. Estos resultados son comparables con el estudio de Del Rio et. al. ${ }^{32}$, quienes encontraron que una disminución de la PTH mayor del $80 \%$ tiene una sensibilidad de $100 \%$ y especificidad del $87 \%$, posiblemente asociado a metodologías

Tabla 5. Valor predictivo de la disminución mayor del $80 \%$ de PTH a las $0,2,4$ y 12 horas versus la presencia de niveles bajos de PTH al 3 mes postoperatorio

\begin{tabular}{lccccccccc}
\hline & \multicolumn{2}{c}{ PTH $>\mathbf{8 0} \%$} & \multicolumn{2}{c}{ PTH $<\mathbf{8 0} \%$} & Sensibilidad & Especificidad & VPP $\%$ & VPN \% & P \\
\cline { 2 - 5 } & $\mathbf{n}$ & $\mathbf{\%}$ & $\mathbf{n}$ & $\mathbf{\%}$ & & & & & \\
\hline PTH 0 horas & 8 & 23,5 & 26 & 76,5 & $60 \%$ & $82,7 \%$ & 37,5 & 92,3 & 0,03 \\
PTH 2 horas & 7 & 20,6 & 27 & 79,4 & $60 \%$ & $86,2 \%$ & 42,8 & 92,5 & 0,01 \\
PTH 4 horas & 4 & 11,8 & 30 & 88,2 & $60 \%$ & $96,5 \%$ & 75 & 93,3 & $<0,001^{*}$ \\
PTH 12 horas & 2 & 5,9 & 32 & 94,1 & $20 \%$ & $96,5 \%$ & 50 & 87,5 & $0,27^{*}$ \\
\hline
\end{tabular}

* Se uso prueba exacta de Fisher 
de investigación similares. Los porcentajes son comparables a los publicados por Gupta ${ }^{30}$ y Kala ${ }^{12}$, con sensibilidad de $100 \%$ y $93 \%$ y especificidad de $87 \%$ y $95 \%$, respectivamente. Tomando diferentes puntos de corte del porcentaje de disminución de PTH, otros autores reportan tasas de sensibilidad y especificidad menores ${ }^{28,29,31}$ para detectar el grupo a riesgo de hipocalcemia posoperatoria; $\sin$ embargo, su histopatología fue predominantemente benigna, en comparación con nuestra población en la cual la mayor parte de las intervenciones fueron realizadas por histopatología maligna.

Entre las limitaciones de este estudio, se encontró la pérdida de pacientes, en quienes no se tomó alguna de las muestras a la hora definida, lo que afectó el tamaño muestral, así como el seguimiento inferior a 6 meses, para definir si la hipocalcemia era permanente. Las fortalezas para resaltar incluyen, la rigurosidad en la toma de las muestras, comparar los dos métodos descritos y usar la PTH para predecir el riesgo de hipocalcemia posterior a tiroidectomía total en el Departamento de Cauca, Colombia.

A pesar de las diferencias reportadas por los grupos de autores, hay acuerdo general acerca de la utilidad de la toma de la PTH postoperatoria para detectar la hipocalcemia posterior a tiroidectomía total. Los resultados de este estudio prospectivo sugieren que una sola medición de PTH menor de $10 \mathrm{pg} / \mathrm{ml}$ o una disminución de la PTH mayor del $80 \%$ del nivel basal, a las 4 horas del postoperatorio, permiten identificar a los pacientes con riesgo de hipocalcemia, y que el segundo método es superior al primero. Con esto se puede lograr una reducción de la morbilidad asociada a la hipocalcemia y el alta temprana de los pacientes, si se considera segura, con o sin suplementación de calcio o calcitriol.

\section{Consideraciones éticas}

Consentimiento informado. Se obtuvo el consentimiento informado de todos los pacientes incluidos. Los Comités de Ética de las instituciones participantes aprobaron el diseño y la metodología del estudio.
Conflicto de intereses. Los autores declararon no tener conflicto de intereses.

Financiamiento. Este trabajo ha sido financiado por los autores.

Contribución de los autores. Concepción y diseño del estudio, adquisición, análisis e interpretación de datos: Jenith Lagos, Jorge A. Herrera.

Redacción del manuscrito, revisión crítica: Daniela Lagos, Ángela Merchán- Galvis.

\section{Referencias}

1. Edafe O, Antakia R, Laskar N, Uttley L, Balasubramanian SP. Systematic review and meta-analysis of predictors of post-thyroidectomy hypocalcaemia: Predictors of post-thyroidectomy hypocalcaemia. Br J Surg. 2014;101:307-20. https://doi.org/10.1002/bjs.9384

2. Saba A, Podda M, Messina-Campanella A, Pisanu A. Early prediction of hypocalcemia following thyroid surgery. A prospective randomized clinical trial. Langenbecks Arch Surg. 2017;402:1119-25. https://doi.org/10.1007/s00423-017-1586-5

3. Cabrera EY, Cifuentes PA, Sanabria Á, Domínguez LC. Tiroidectomía ambulatoria: análisis de minimización de costos en Colombia. Rev Colomb Cir. 2014;29: 319-26.

4. Calvo-Espino P, Rivera-Bautista JÁ, Artés-Caselles M, Serrano-González J, García-Pavía A, García-Oria MJ, et al. Uso del valor de la hormona paratiroidea intacta en el primer día postoperatorio tras tiroidectomía total como predictor de hipoparatiroidismo permanente. Endocrinol Diabetes Nutr. 2019;66:195-201. https://doi.org/10.1016/j.endinu.2018.08.006

5. Dedivitis RA, Aires FT, Cernea CR. Hypoparathyroidism after thyroidectomy: prevention, assessment and management. Curr Opin Otolaryngol Head Neck Surg. 2017;25:142-6. https://doi.org/10.1097/M00.0000000000000346

6. Ravikumar K, Sadacharan D, Muthukumar S, Sundarram T, Periyasamy S, Suresh R. A prospective study on role of supplemental oral calcium and Vitamin D in prevention of postthyroidectomy hypocalcemia. Indian J Endocrinol Metab. 2017;21:498-503. https://doi.org/10.4103/ijem.IJEM_402_16

7. Mordojovich G, Lavín M, Ávalos N. medición de niveles de parathormona como predictor de hipocalcemia sintomática en el post-operatorio precoz de tiroidectomía total. Rev Chil Cir. 2015;67:147-52.

https://doi.org/10.4067/S0718-40262015000200006 
8. Wang X, Zhu J, Liu F, Gong Y, Li Z. Postoperative hypomagnesaemia is not associated with hypocalcemia in thyroid cancer patients undergoing total thyroidectomy plus central compartment neck dissection. Int J Surg. 2017;39:192-6. https://doi.org/10.1016/j.ijsu.2017.01.085

9. Rosa KM, de Matos LL, Cernea CR, Brandão LG, de Araújo Filho VJF. Postoperative calcium levels as a diagnostic measure for hypoparathyroidism after total thyroidectomy. Arch Endocrinol Metab. 2015;59:428-33. https://doi.org/10.1590/2359-3997000000074

10. Noureldine SI, Genther DJ, Lopez M, Agrawal N, Tufano RP. Early predictors of hypocalcemia after total thyroidectomy: An analysis of 304 patients using a shortstay monitoring protocol. JAMA Otolaryngol Neck Surg. 2014;140:1006-13. https://doi.org/10.1001/jamaoto.2014.2435

11. Chisthi MM, Nair RS, Kuttanchettiyar KG, Yadev I. Mechanisms behind post-thyroidectomy hypocalcemia: Interplay of calcitonin, parathormone, and albumin - A prospective study. J Invest Surg. 2017;30:217-25. https://doi.org/10.1080/08941939.2016.1235238

12. Kala F, Sarici IS, Ulutas KT, Sevim Y, Dogu A, Sarigoz T, et al. Intact parathormone measurement 1 hour after total thyroidectomy as a predictor of symptomatic hypocalcemia. Int J Clin Exp Med. 2015;8:18813-8.

13. Eismontas V, Slepavicius A, Janusonis V, Zeromskas $P$, Beisa V, Strupas K, et al. Predictors of postoperative hypocalcemia occurring after a total thyroidectomy: results of prospective multicenter study. BMC Surg. 2018;18:55.

https://doi.org/10.1186/s12893-018-0387-2

14. Haden ST, Brown EM, Hurwitz S, Scott J, Fuleihan GEH. The effects of age and gender on parathyroid hormone dynamics. Clin Endocrinol (Oxf). 2000;52:329-38. https://doi.org/10.1046/j.1365-2265.2000.00912.x

15. Brown EM. Four-parameter model of the sigmoidal relationship between parathyroid hormone release and extracellular calcium concentration in normal and abnormal parathyroid tissue. J Clin Endocrinol Metab. 1983;56:572-81.

https://doi.org/10.1210/jcem-56-3-572

16. Quiros RM, Pesce CE, Wilhelm SM, Djuricin G, Prinz RA. Intraoperative parathyroid hormone levels in thyroid surgery are predictive of postoperative hypoparathyroidism and need for vitamin D supplementation. Am J Surg. 2005;189:306-9. https://doi.org/10.1016/j.amjsurg.2005.01.006

17. Lindblom P, Westerdahl J, Bergenfelz A. Low parathyroid hormone levels after thyroid surgery: A feasible predictor of hypocalcemia. Surgery. 2002;131:515-20. https://doi.org/10.1067/msy.2002.123005

18. Lombardi CP, Raffaelli M, Princi P, Santini S, Boscherini $\mathrm{M}$, De Crea C, et al. Early prediction of postthyroidec- tomy hypocalcemia by one single iPTH measurement. Surgery. 2004;136:1236-41.

https://doi.org/10.1016/j.surg.2004.06.053

19. Lombardi CP, Raffaelli M, Princi P, Dobrinja C, Carrozza C, Di Stasio E, et al. Parathyroid hormone levels 4 hours after surgery do not accurately predict post-thyroidectomy hypocalcemia. Surgery. 2006;140:1016-25. https://doi.org/10.1016/j.surg.2006.08.009

20. Australian Endocrine Surgeons Guidelines AES06/01. Postoperative parathyroid hormone measurement and early discharge after total thyroidectomy: Analysis of Australian data and management recommendations. ANZ J Surg. 2007;77:199-202. https://doi.org/10.1111/j.1445-2197.2007.04018.x

21. Landry CS, Grubbs EG, Hernandez M, Hu MI, Hansen MO, Lee JE, Perrier ND. Predictable criteria for selective, rather than routine, calcium supplementation following thyroidectomy. Arch Surg. 2012;147:338-44. https://doi.org/10.1001/archsurg.2011.1406

22. Cayo AK, Yen TWF, Misustin SM, Wall K, Wilson SD, Evans DB, Wang TS. Predicting the need for calcium and calcitriol supplementation after total thyroidectomy: Results of a prospective, randomized study. Surgery. 2012;152:1059-67. https://doi.org/10.1016/j.surg.2012.08.030

23. Grodski S, Serpell J. Evidence for the role of perioperative PTH measurement after total thyroidectomy as a predictor of hypocalcemia. World J Surg. 2008;32:136773. https://doi.org/10.1007/s00268-008-9545-5

24. Grodski S, Farrell S. Early postoperative PTH levels as a predictor of hypocalcaemia and facilitating safe early discharge after total thyroidectomy. Asian J Surg. 2007;30:178-82. https://doi.org/10.1016/S1015-9584(08)60019-6

25. Carr AA, Yen TW, Fareau GG, Cayo AK, Misustin SM, Evans DB, Wang TS. A single parathyroid hormone level obtained 4 hours after total thyroidectomy predicts the need for postoperative calcium supplementation. J Am Coll Surg. 2014;219:757-64. https://doi.org/10.1016/j.jamcollsurg.2014.06.003

26. Sywak MS, Palazzo FF, Yeh M, Wilkinson M, Snook K, Sidhu SB, Delbridge LW. Parathyroid hormone assay predicts hypocalcaemia after total thyroidectomy. ANZ J Surg. 2007;77:667-70. https://doi.org/10.1111/j.1445-2197.2007.04183.x

27. AlQahtani A, Parsyan A, Payne R, Tabah R. Parathyroid hormone levels 1 hour after thyroidectomy: an early predictor of postoperative hypocalcemia. Can J Surg. 2014;57:237-40. https://doi.org/10.1503/cjs.008013

28. Sahli Z, Najafian A, Kahan S, Schneider EB, Zeiger MA, Mathur A. One-hour postoperative parathyroid hormone levels do not reliably predict hypocalcemia after thyroidectomy. World J Surg. 2018;42:2128-33. https://doi.org/10.1007/s00268-017-4444-2 
29. Cannizzaro MA, Okatyeva V, Lo Bianco S, Caruso V, Buffone A. Hypocalcemia after thyroidectomy: iPTH levels and iPTH decline are predictive? Retrospective cohort study. Ann Med Surg. 2018;30:42-5. https://doi.org/10.1016/j.amsu.2018.04.032

30. Gupta S, Chaudhary P, Durga CK, Naskar D. Validation of intra-operative parathyroid hormone and its decline as early predictors of hypoparathyroidism after total thyroidectomy: A prospective cohort study. Int J Surg. 2015;18:150-3.

https://doi.org/10.1016/j.ijsu.2015.04.074

31. Karatzanis AD, Ierodiakonou DP, Fountakis ES, Velegrakis SG, Doulaptsi MV, Prokopakis EP, et al. Postoperative day 1 levels of parathyroid as predictor of occurrence and severity of hypocalcaemia after total thyroidectomy. Head Neck. 2018;40:1040-5.

https://doi.org/10.1002/hed.25081
32. Del Rio L, Castro A, Bernáldez R, Del Palacio A, Giráldez $\mathrm{CV}$, Lecumberri B, et al. Valor predictivo de la paratohormona en la hipocalcemia postiroidectomía. Acta Otorrinolaringologica. 2011;62:265-73. https://doi.org/10.1016/j.otoeng.2011.01.002

33. Ferlay J, Soerjomataram I, Dikshit R, Eser S, Mathers C, Rebelo M, et al. Cancer Incidence and Mortality Worldwide: sources, methods and major patterns in GLOBOCAN 2012. Int J Cancer. 2015;136:E359-86. https://doi.org/10.1002/ijc.29210

34. Falch C, Hornig J, Senne M, Braun M, Konigsrainer A, Kirschniak A, Muller S. Factors predicting hypocalcemia after total thyroidectomy - A retrospective cohort analysis. Int J Surg. 2018;55:46-50.

https://doi.org/10.1016/j.ijsu.2018.05.014 